\title{
Dog10K_Boxer_Tasha_1.0: A Long-Read Assembly of the Dog Reference Genome
}

\author{
Vidhya Jagannathan ${ }^{1}$, Christophe Hitte ${ }^{2}$, Jeffrey M. Kidd ${ }^{3,4}$, Patrick Masterson ${ }^{5}$, Terence D. Murphy ${ }^{5}$, \\ Sarah Emery ${ }^{3}$, Brian Davis ${ }^{6}$, Reuben M. Buckley ${ }^{7}$, Yan-Hu Liu ${ }^{8,9}$, Xiang-Quan Zhang ${ }^{8,9}$, Tosso Leeb ${ }^{1}$, \\ Ya-Ping Zhang ${ }^{8,9}$, Elaine A. Ostrander ${ }^{7}$ and Guo-Dong Wang ${ }^{8,9, *}$
}

Citation: Jagannathan, V.; Hitte, C. Kidd, J.M.; Masterson, P.; Murphy, T.D.; Emery, S.; Davis, B.; Buckley, R.M.; Liu, Y.-H.; Zhang, X.-Q.; et al. Dog10K_Boxer_Tasha_1.0: A LongRead Assembly of the Dog Reference Genome. Genes 2021, 12, 847. https://doi.org/10.3390/ genes 12060847

Academic Editor: Benjamin N. Sacks

Received: 5 May 2021

Accepted: 27 May 2021

Published: 30 May 2021

Publisher's Note: MDPI stays neutral with regard to jurisdictional claims in published maps and institutional affiliations.

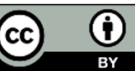

Copyright: $(2021$ by the authors. Licensee MDPI, Basel, Switzerland. This article is an open access article distributed under the terms and conditions of the Creative Commons Attribution (CC BY) license (http://creativecommons.org/licenses/by/4.0/).
1 Vetsuisse Faculty, Institute of Genetics, University of Bern, 3001 Bern, Switzerland; vidhya.jagannathan@vetsuisse.unibe.ch (V.J.); tosso.leeb@vetsuisse.unibe.ch (T.L.)

2 Institute Genetics Development Rennes, University of Rennes, CNRS-UMR 6290, F-35000 Rennes, France; hitte@univ-rennes1.fr

3 Department of Human Genetics, University of Michigan, Ann Arbor, MI 48109, USA; jmkidd@umich.edu (J.M.K.); sbherman@umich.edu (S.E.)

4 Department of Computational Medicine and Bioinformatics, University of Michigan Medical School, Ann Arbor, MI 48109, USA

5 National Center for Biotechnology Information, National Library of Medicine, National Institutes of Health, Bethesda, MA 20894, USA; patrick.masterson@nih.gov (P.M.); murphyte@nih.gov (T.D.M.)

6 Department of Integrative Biological Sciences, Texas A and M University, College Station, TX 77840, USA; bdavis@cvm.tamu.edu

7 National Human Genome Research Institute, National Institutes of Health, Bethesda, MA 20894, USA; reuben.buckley@nih.gov (R.M.B.); eostrand@mail.nih.gov (E.A.O.)

8 State Key Laboratory of Genetic Resources and Evolution, Kunming Institute of Zoology, Chinese Academy of Sciences, Kunming 650201, China; liuyanhu@mail.kiz.ac.cn (Y.-H.L.); zhangxqkiz@163.com (X.-Q.Z.); zhangyp@mail.kiz.ac.cn (Y.-P.Z.)

9 Center for Excellence in Animal Evolution and Genetics, Chinese Academy of Sciences, Kunming 650201, China

* Correspondence: wanggd@mail.kiz.ac.cn

\begin{abstract}
The domestic dog has evolved to be an important biomedical model for studies regarding the genetic basis of disease, morphology and behavior. Genetic studies in the dog have relied on a draft reference genome of a purebred female boxer dog named "Tasha" initially published in 2005. Derived from a Sanger whole genome shotgun sequencing approach coupled with limited clonebased sequencing, the initial assembly and subsequent updates have served as the predominant resource for canine genetics for 15 years. While the initial assembly produced a good-quality draft, as with all assemblies produced at the time, it contained gaps, assembly errors and missing sequences, particularly in GC-rich regions, which are found at many promoters and in the first exons of protein-coding genes. Here, we present Dog10K_Boxer_Tasha_1.0, an improved chromosomelevel highly contiguous genome assembly of Tasha created with long-read technologies that increases sequence contiguity >100-fold, closes $>23,000$ gaps of the CanFam3.1 reference assembly and improves gene annotation by identifying $>1200$ new protein-coding transcripts. The assembly and annotation are available at NCBI under the accession GCF_000002285.5.
\end{abstract}

Keywords: Canis lupus familiaris; high quality; contiguity; Pacific Biosciences; annotation; resource

\section{Introduction}

High-quality reference genomes are fundamental assets for the study of genetic variation in any species. The ability to link genotype to phenotype and the subsequent identification of functional variants rely on high fidelity assessment of variants throughout the genome. This reliance is well illustrated by the domestic dog, which offers specific challenges for any genetic study. Featuring over 350 pure breeding populations, each breed is a mosaic of ancient and modern variants, and each reflects a complex history linking it to 
other related breeds. As a result of bottlenecks associated with domestication $(15,000$ 30,000 years before present) and more recent individual breed formation (50-250 years before present), dog genomes contain long and frequent stretches of linkage disequilibrium (LD). While helpful for identifying loci of interest, long LD makes the necessary fine mapping for moving from marker to gene to variant both labor-intensive and error-prone.

In 2005, the first high-quality draft (7.5×) sequence of a Boxer dog, named Tasha, was made publicly available [1]. The reference sequence has proven useful in discoveries of canine-associated molecular variants [2,3], including single-nucleotide variants (SNVs) and small indels, regulatory sequences $[4,5]$, large rearrangements and copy number variants [6,7] associated with both inter and intra gene variation. The resulting SNV arrays, designed based on variation relative to the Tasha-derived assembly, have led to the success of hundreds of genome-wide association studies (GWAS), advancing the dog as a system for studies of disease susceptibility and molecular pathomechanisms, evolution, and behavior. However, for the dog system to advance further, long-read high-quality assemblies from different individuals are needed. This will greatly improve the sensitivity of variant detection, especially for large structural variation. Furthermore, high-quality assemblies are an essential prerequisite for accurate annotation, which is required to assay the potential functional effects of detected variants. Recently, several high-quality genomes from different dogs became available [8-10].

However, using the same dog as used for the initial assembly offers specific advantages, including the ability to integrate new findings with previous observations. A high-quality genome assembly from the boxer Tasha will mean that the value of existing resources, such as existing bacterial artificial chromosome (BAC) libraries, and the wealth of experience and knowledge gained using previous versions of this dog's genome, will be preserved for future research efforts. The dog genome assembly reported here was built using a combination of Pacific Biosciences (PacBio) continuous long-read (CLR) sequencing technology, 10x Chromium-linked reads, BAC pair-end sequences and the draft reference genome sequence CanFam3.1.

\section{Materials and Methods}

\subsection{Whole Genome Sequencing}

A single blood draw from which genomic DNA was isolated from blood leukocytes of a female Boxer, Tasha, and which was also used to generate the previous CanFam 1, CanFam 2 and CanFam 3 genome assemblies, was utilized here. Continuous long-read (CLR) sequencing was carried out at Novogene Bioinformatics Technology Co., Ltd. (Beijing, China) with a PacBio Sequel sequencer (Pacific Biosciences, Menlo Park, CA, USA). Approximately $100 \mu \mathrm{g}$ of genomic DNA were used for sequencing. SMRTbell libraries were prepared using a DNA Template Prep Kit 1.0 (PacBio), and 56 20-kb SMRTbell libraries were constructed. A total of $252 \mathrm{~Gb}$ of sequence data were collected. High molecular weight DNA from Tasha was also sequenced with Chromium libraries (10x Genomics, Pleasanton, CA, USA) on Illumina (San Diego, CA, USA) HiSeq X $(2 \times 150 \mathrm{bp})$, generating $589,824,390$ read pairs or $176 \mathrm{~Gb}$ of data.

\subsection{Genome Assembly Workflow}

We assembled the genome using the Canu (v1.6) [11] and wtdbg2 [12] assembly algorithms. Briefly, the pipeline was composed of assembly, scaffolding and a final polishing step. PacBio reads had a mean read length of $8.5 \mathrm{~kb}$ and were used for the de novo assembly. The reads were corrected using the Canu error correction module, which generates a consensus sequence for each read using its best set of long read overlaps. The corrected consensus reads were then assembled using the wtdbg2 algorithm [12], which is designed for assembly of long reads produced by the PacBio or Nanopore technologies. The assembled contigs were polished with raw PacBio reads using the WTPOA-CNS tool of the WTDBG2 package. This was followed by misassembly detection and correction 
with TIGMINT [13]. End sequences from BAC clones were extracted from the TraceDB of NCBI and used for scaffolding corrected contigs using the BESST algorithm (v2.2.8) [14]. Gap filling was performed using the PacBio subreads with PBjelly (from PBSuite v15.8.24) [15] and one additional round of genome polishing was carried out using Pilon v1.23. [16] with the 10x Chromium reads. Finally, RaGOO (v1.1) [17] was used for reference-guided scaffolding, using CanFam3.1 as the reference. The draft scaffolds were subjected to additional gap closure using PBJelly.

\subsection{Assembly Quality Control}

The scaffold order and orientation of the assembly was assessed by aligning it to an existing radiation hybrid map (RH-map) comprising 10,000 markers [18]. A chromosomewide review of scaffold discrepancies was determined visually, by aligning the sequences of RH map markers against the assembled scaffolds. The generated dot plots were examined for contigs that were incorrectly ordered in scaffolds and these were manually inspected and eventually reordered. The assembly was also assessed for completeness using BUSCO [19], which provides a summary of genome completeness using a database of expected gene content based on near-universal single-copy orthologs from mammalian species with genomic sequence data. This includes 4104 single copy genes that are evolutionarily conserved between mammals.

\subsubsection{Fosmid End Sequence Alignment}

End sequences from previously constructed fosmid libraries from Tasha were aligned to the assembly as previously described [1]. Concordant clones were considered to be those with an inward read orientation and a size between 35,328 and $43,453 \mathrm{bp}$. Using bedtools [20], the physical coverage of concordant clones in $5 \mathrm{~kb}$ windows along the genome was determined. Segments of the primary chromosome assemblies that were not supported by any concordant fosmids were also identified. Analysis was limited to the primary chromosome assemblies (chr1-chr38, chrX) and any interval that intersected with chromosome ends was discarded. This resulted in a total of 1004 regions, of which 282 intersected with a segmental duplication interval (considering the union of assembly and read-depth-based annotations). To assess the significance of the intersection with segmental duplications, we performed 1000 random permutations of the intervals using bedtools and found that 49 to 103 of the intervals intersected with a duplication, with a mean intersection rate of 75 .

\subsubsection{Alignment of Finished BAC Clone Sequences}

A list of assembled BAC clones from the $\mathrm{CH}-82$ library was obtained from ftp://ftp.ncbi.nih.gov/repository/clone/reports/Canis_familiaris/CH82.clone_ac-

state_9615.out (accessed on 26 June 2019). The sequence of 395 finished clones was aligned to the long-read Tasha assembly using minimap2 (v2.17) [21]. One clone (AC190394.3) did not have a minimap2 alignment, 124 clones returned multiple alignment positions, 124 clones aligned to a single position annotated as duplicated in the Dog_10K_Boxer_Tasha_1.0 assembly, and four clones returned alignments that did not include the entire BAC sequence. We therefore focused on a set of 142 clones that had alignment to a single locus based on minimap2 with a query alignment that encompassed the entire clone length and that did not overlap with regions annotated as segmental duplications in the Dog_10K_Boxer_Tasha_1.0 assembly. An optimal global sequence alignment between the BAC sequence and the assembly was then determined using a stretcher [22] with default parameters. 


\subsection{Detection of Common Repeats and Segmental Duplications}

Common repeats were identified with RepeatMasker (v4.0.7) using the rmblastn (v2.2.27+) search engine and a combined repeat database consisting of the Dfam_Consensus-20170127 [23] and RepBase-20170127 [24] releases.

Segmental duplications in the assembly were detected using two approaches. First, duplicated regions were identified based on assembly self-alignment using the program SEDEF [25]. Duplications with at least $90 \%$ sequence identity and length of $1 \mathrm{~kb}$ were retained. Second, duplications were defined based on an analysis of the depth of coverage of Illumina sequencing data using the fastCN [26] program. Copy number was estimated in non-overlapping windows each containing $3 \mathrm{kbp}$ of unmasked sequence. Control regions for normalization were converted to Dog10K_Boxer_Tasha_1.0 coordinates using the liftOver tool $[27,28]$. Segmental duplications were defined as segments of four or more consecutive windows with an estimated copy number of at least 2.5. Comparable annotations for the CanFam3.1 assembly were obtained from [8].

\subsection{Gene Annotation}

The assembly was annotated using the previously described NCBI pipeline [29,30]. The pipeline uses a WindowMasker-masked genome for building gene models substantiated with RNA-seq data and protein alignments. RNA-sequencing data from various dog tissues were used for gene prediction (https://www.ncbi.nlm.nih.gov/genome/annotation_euk/Canis_lupus_familiaris/106/) (accessed on 1st Feb 2021).

\subsection{Genome Assembly Alignment}

The Dog10K_Boxer_Tasha_1.0 assembly was aligned to the CanFam3.1 assembly using minimap2 (v2.17) [21] with the 'asm5' option. Insertions and deletions were identified using the paftools.js program distributed with minimap2 with default options. Analysis was restricted to the primary chromosome sequences (chr1-38 and chrX). Regions that overlapped with assembly gaps, segmental duplications detected based on assembly selfalignment, or segmental duplications identified by read depth were removed.

\subsection{Structural Variant Detection}

Raw PacBio reads were aligned to the CanFam3.1 and Dog10K_Boxer_Tasha_1.0 assemblies using minimap2 (v2.17) [21]. Structural variants were identified using sniffles (v1.0.12) [31]. Only calls with precise breakpoints on the primary chromosome sequences (chr1-38 and chrX) were considered. Calls were filtered to remove insertions and deletions that intersect with assembly gaps.

\subsection{BAC Assembly}

Bacterial artificial chromosome (BAC) clones that mapped to the amylase locus were received from the $\mathrm{BACPAC}$ resources center (Emeryville, $\mathrm{CA}, \mathrm{USA}$ ). BACs were streaked to obtain single clones on $\mathrm{LB}$ agar with $100 \mathrm{ug} / \mathrm{ul}$ chloramphenicol and singe clones were cultured $20-24 \mathrm{~h}$ at $37^{\circ} \mathrm{C}$ in $100 \mathrm{~mL}$ LB broth with $100 \mathrm{ug} / \mathrm{uL}$ chloramphenicol. BAC DNA was isolated using NucleoBond Xtra Midi kit for transfection-grade plasmid DNA without NucleoBond ${ }^{\circledR}$ Finalizer (Machery-Nagel, Bethlehem, PA, USA) and, after precipitation and drying, resuspended in $500 \mathrm{uL} \mathrm{H}_{2} \mathrm{O}$ by incubating $72-96 \mathrm{~h}$ at $4{ }^{\circ} \mathrm{C}$. Within $48 \mathrm{~h}$ of resuspension, BAC DNA was sequenced on a Minion with the Flongle adapter (Oxford Nanopore Technologies, Oxford, UK). Libraries were made using the Rapid Barcoding Sequencing kit (Oxford Nanopore Technologies, SQK-RBK004) according to the manufacturer's protocol, except for fragmentation where $0.25 \mathrm{uL}$ of Fragmentation Mix was mixed with $200 \mathrm{ng}$ of DNA in $4.75 \mathrm{uL}$ of water, incubated $30^{\circ} \mathrm{C}$ for $1 \mathrm{~min}$ then $80^{\circ} \mathrm{C}$ for $1 \mathrm{~min}$, and cooled on ice. Following fragmentation, BAC libraries were pooled by adding $1.67 \mathrm{uL}$ of each library prep; 0.5 uL Rapid Primer (RAP) was added, and the mix was incubated 
for $5 \mathrm{~min}$ at room temperature. Flow cells were primed and loaded according to the manufacturer's protocol.

Nanopore reads from the BAC were assembled using the pipeline described in https://github.com/KiddLab/run_canu_bac (accessed on 1st Feb 2021). Briefly, raw reads were filtered for hits of Escherichia coli and assembled using canu (v2.1) [11]. The unique portion of the resulting circular contig was then extracted and polished using racon (v1.4.10) [32]. Finally, the vector backbone sequence was removed and the contig was rotated to begin at the appropriate position. The final $\mathrm{CH} 82-451 \mathrm{P03}$ sequence was compared to the Dog10K_Boxer_Tasha_1.0 assembly using MUMmer (v3.23) [33].

\subsection{Mapping SNV Array Probes}

Chromosomal sequences from CanFam3.1 and Dog10K_Boxer_Tasha_1.0 were aligned to each other using blat [34]. The aligned fragments were processed using UCSC tools to create the necessary chain file for use with the liftOver tool. The liftOver was performed using the default settings with the "-multiple" option included. Genomic positions from both the Affymetrix (Santa Clara, CA, USA) Axiom Canine HD Array and Illumina (San Diego, CA, USA) CanineHD BeadChip were converted from CanFam3.1 to Dog10K_Boxer_Tasha_1.0. Genomic positions were obtained for the Axiom Canine HD Array from: https://sec-assets.thermofisher.com/TFS-Assets/LSG/Support-Files/Axiom_K9_HD.na35.r5.a7.annot.csv.zip (accessed on 21st Nov 2020); and for the CanineHD BeadChip: ftp://webdata2:webdata2@ussd-ftp.illumina.com/downloads/ProductFiles/CanineHD/CanineHD_B.csv (accessed on 21st Nov 2020). The bed files resulting from the lift over were converted to Plink map files. All markers were included in each map file and markers were ordered sequentially according to the order they were downloaded from their corresponding URLs. Markers for which no position was obtained were placed on chromosome " 0 " at position " 0 ".

\section{Results}

DNA isolated and stored at $-80{ }^{\circ} \mathrm{C}$ at NHGRI from the same female Boxer, Tasha, used for the CanFam $3.1 \mathrm{draft}$ genome sequence was utilized to generate a new assembly. Frozen DNA from the same aliquot was thawed and used to prepare high molecular weight DNA libraries, which were sequenced using PacBio single-molecule real-time (SMRT) and 10x Genomics Linked-Reads sequencing technologies. Approximately 100fold coverage $(252 \mathrm{~Gb})$ and 74 -fold coverage $(176 \mathrm{~Gb})$ of the genome were generated using PacBio and 10x Genomics reads, respectively.

\subsection{Dog10K_Boxer_Tasha_1.0 Assembly}

PacBio SMRT cells produced 27,878,642 reads with a mean length of $8514 \mathrm{bp}$ and N50 read length - a length at which $50 \%$ of the bases are in reads longer or equal to-was 13,189 bp. All PacBio reads were used for the assembly. The assembly pipeline (Figure 1) underwent initial read correction with Canu. After correction, 558,6195 reads were used for assembling with wtdbg2, obtaining a corrected read cut-off of $14 \mathrm{~kb}$ that provided 43fold $(104,569,563,638$ bases) genome coverage for input. The initial ungapped assembly of WTDBG2 contained 1562 contigs with an N50 of $23.8 \mathrm{Mb}$. Tigmint (v.0.4) was used to correct initial assembly errors by incorporating the linked reads generated by $10 x \mathrm{Ge}-$ nomics Chromium long-read technology. Tigmint split 75 misassembled contigs, which resulted in an assembly featuring 1786 contigs, of which 1724 were $>500 \mathrm{bp}$. The assembly contig N50-the contig length in the assembly where equal or longer contigs contain half the bases of the genome-was $23.72 \mathrm{Mb}$.

The Tigmint-corrected assembly was then scaffolded with BAC end sequences. The resultant scaffolding, constructed with the BESST algorithm (v 2.2.8), resulted in an assembly of 1685 scaffolds, which increased the N50 to $27.4 \mathrm{Mb}$. RaGOO was then used to 
scaffold the data into 39 chromosomes based on CanFam3.1. The chromosome level scaffolds had a minimum of four contigs as noted on chromosomes 28,30 and 36 and a maximum of 82 contigs on the X chromosome. The N50 of the scaffolded assembly was $63,738,581 \mathrm{bp}$ (Table 1). The assembly contained 621 spanned gaps closing $>23,000$ of the CanFam3.1 assembly (18.25 Mb) (Figure 1). The number of unplaced scaffolds was 107 with an average length of $19.8 \mathrm{~kb}$ and consisting of 2,127,951 bases.

Table 1. Summary statistics for the Dog10K_Boxer_Tasha_1.0 genome assembly and comparison with current dog reference genome CanFam3.1.

\begin{tabular}{ccc}
\hline Statistic & CanFam3.1 & Dog10K_Boxer_Tasha_1.0 \\
\hline Total sequence length & $2,410,976,875$ & $2,312,802,206$ \\
\hline Total ungapped length & $2,392,715,236$ & $2,312,743,367$ \\
\hline No. of scaffolds & 3310 & 147 \\
\hline No. of unplaced scaffolds & 3228 & 107 \\
\hline Scaffold N50 & $45,876,610$ & $63,738,581$ \\
\hline Scaffold L50 & 20 & 14 \\
\hline No. of unspanned gaps & 80 & 399 \\
\hline No. of spanned gaps & 23,796 & 621 \\
\hline No. of contigs & 27,106 & 1162 \\
\hline Contig N50 & 267,478 & $27,487,084$ \\
\hline Contig L50 & 2436 & 31 \\
\hline No. of chromosomes & 39 & 39
\end{tabular}

The quality of the Dog10K_Boxer_Tasha_1.0 assembly was assessed by comparison with an existing RH-map of 10,000 markers. The comparison strongly supported the overall accuracy of the assembly. There were two major discordances between the RH map and the draft assembly order of the contigs, one on chromosome 6 and the other on chromosome 11. The order was corrected, and gaps were again closed using PBJelly and PacBio SMRT raw reads. Discrepancies involving blocks of $\sim 1 \mathrm{Mb}$ on chromosome 9 and 0.2 $\mathrm{Mb}$ on chromosome 16 could not be resolved and will require further investigation. 


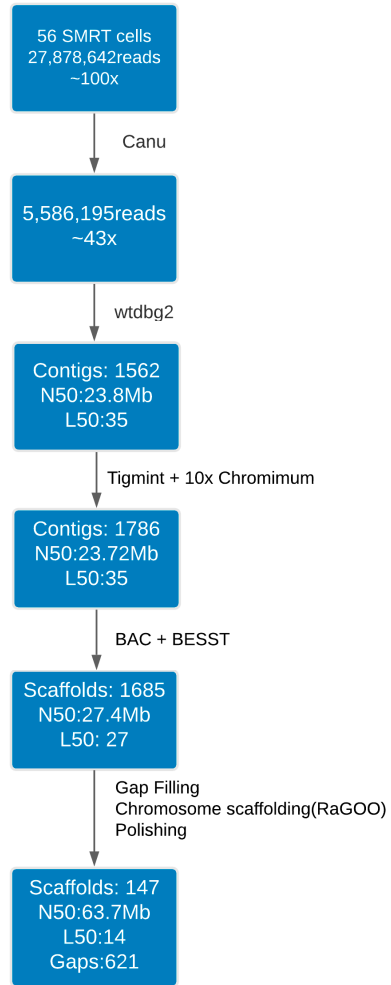

(a)

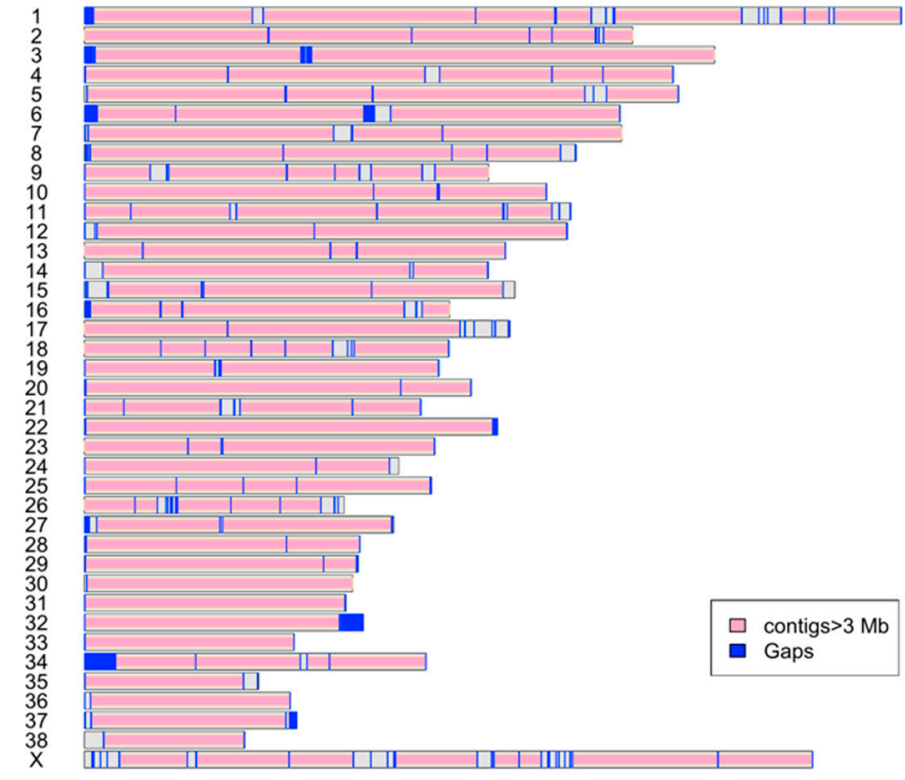

(b)

Figure 1. Dog_10k_Boxer_Tasha_1.0 assembly. (a) Assembly workflow pipeline. The different algorithms used in the pipeline have been indicated. N50 is the contig/scaffold length in the assembly where equal or longer contigs contain $50 \%$ of the genome. L50 count is the number of contigs whose length sum makes N50. (b) Ideogram showing chromosomes, contigs, and gaps. The grey regions indicate contigs of size less than $3 \mathrm{Mb}$.

\subsection{Assembly Quality Assessment}

We used fosmid clone end sequences to identify regions that may be misassembled in Dog_10k_Boxer_Tasha_1.0. We identified 895,746 clones with a concordant mapping based on the orientation of the end-sequences and the apparent size of the cloned fragment (Figure S1), yielding a median genomic physical coverage of 17 concordant clones (Figure S2). Using this map of fosmid coverage, we identified 1004 intervals (32.5 Mb) on the primary chromosomes that do not intersect with a concordantly mapping fosmid (Table S1). We found that 282 of these intervals intersected with regions of segmental duplication in Dog_10K_Boxer_Tasha_1.0, a value greater than that observed in any of 1000 random permutations. This indicates that duplicated regions are enriched for potential misassembly.

We also assessed the per-bp sequence accuracy of the Dog_10k_Boxer_Tasha_1.0 assembly using 142 finished BAC clones from the $\mathrm{CH}-82$ library that have a unique alignment to Dog_10k_Boxer_Tasha_1.0. Discarding alignment gaps, mismatches were observed at 14,255 of 26,778,153 aligned nucleotides (Figure S3). Assuming that all mismatches represent errors in the Dog_10k_Boxer_Tasha_1.0 sequence-a conservative assumption since heterozygous sites as well as errors in the BAC sequence are expectedthe observed mismatch rate corresponds to an estimated per-base sequence quality [35] of Q33. We note, however, that the apparent number of alignment gaps is higher than the apparent single base substitution rate, suggesting that indels remain the primary error mode in long-read assemblies (Table S2). 


\subsection{Assembly Completeness}

The completeness of the assembly was assessed using BUSCO, which uses a set of universal single-copy orthologs. This analysis showed an improvement of BUSCO completeness from 92.2\% in CanFam3.1 to 95.3\% in Dog10K_Boxer_Tasha_1.0 (Table 2).

Table 2. Comparison of BUSCO analysis of genomes.

\begin{tabular}{ccc}
\hline Statistic & Dog10k_Boxer_Tasha_1.0 & CanFam3.1 \\
\hline Complete BUSCOs & $95.3 \%$ & $92.2 \%$ \\
\hline Complete and single copy BUSCOs & $94.1 \%$ & $91.1 \%$ \\
\hline Complete and duplicated BUSCOs & $1.2 \%$ & $1.1 \%$ \\
\hline Fragmented BUSCOs & $2.1 \%$ & $4.0 \%$ \\
\hline Missing BUSCOs & $2.6 \%$ & $3.8 \%$ \\
\hline
\end{tabular}

We further compared the structural accuracy of the RaGOO arranged chromosomelevel scaffolds to that of the CanFam3.1 chromosomes. We identified several regions known to be misassembled in CanFam3.1 and were now corrected in the Dog10k_Boxer_Tasha_1.0 assembly. These regions were supported by corresponding BAC end sequences (Figure S4).

Additionally, the orientation of chromosomes 27 and 32 was reversed compared to CanFam3.1. The two chromosomal re-orientations were backed by evidence in [36] and [37], based on recombination rates in dog chromosomes and fluorescence in situ hybridization experiments by Matthew Breen (personal communication).

\subsection{Gene Annotation}

Annotation of the Dog10K_Boxer_Tasha_1.0 assembly was carried out using the NCBI annotation pipeline and released via the NCBI ftp site [38]. The annotation pipeline used RNA-seq data from more than 25 tissues, along with known RefSeq, Genbank transcripts and canine expressed sequence tags. Statistics from the annotation release 106 are listed in Table 3. The annotation includes 20,100 protein-coding genes, which is comparable to annotations of other carnivores (average 20,105, stdev 1078, from 27 species). A total of 1299 protein-coding transcripts from 737 genes were identified as novel as they do not align to CanFam3.1 assembly. We found 78 out of 2473 known RefSeq transcripts did not map to the Dog10k_Boxer_Tasha_1.0 assembly [38]. Significantly, we observed a 7.0\% increase $(17,721$ vs. 16,554$)$ in the number of annotated protein-coding genes with very high coverage ( $\geq 90 \%$ ) alignments compared to their best hits in SwissProt, with $88 \%$ of all protein-coding genes having at least one isoform exceeding $90 \%$ coverage. In addition, the new Tasha assembly has only $4.5 \%$ (891) of protein-coding genes represented with corrected models that compensate for suspected frameshifts or premature stop codons in the genome, compared to $5.5 \%$ for the prior NCBI annotation of CanFam3.1, or 5.6-11.3\% for NCBI annotations of several other canine assemblies. These improvements can be largely attributed to fewer assembly gaps and the fact that gaps comprising exons of several genes have now been closed (Figure S5). For example, 5770 genes in CanFam3.1 have gaps within and flanking them. Only 12 of these genes still have gaps overlapping their exons and introns in Dog_10k_Boxer_Tasha_1.0. 
Table 3. Annotation statistics for NCBI annotation release $106 .{ }^{*}$ are non-coding RNA genes that cannot be classified.

\begin{tabular}{cc}
\hline Feature & $\begin{array}{c}\text { Dog10k_Boxer_Tasha_1.0/Annotation } \\
\text { Release 106 }\end{array}$ \\
\hline Protein-coding genes & 20,100 \\
\hline Non-coding genes & 15,306 \\
\hline Small non-coding genes & 2083 \\
\hline Long non-coding genes & 12,667 \\
\hline Miscellaneous $^{*}$ non-coding genes & 10 \\
\hline Pseudogenes & 4887 \\
\hline
\end{tabular}

\subsection{SNV Array Probes Mapped to Dog10k_Boxer_Tasha_1.0}

Marker positions from the Axiom Canine HD Array and CanineHD BeadChip were mapped from CanFam3.1 to Dog10K_Boxer_Tasha_1.0. For the Axiom Canine HD Array and CanineHD BeadChip, $98.12 \%$ and $97.91 \%$ of markers, respectively, were successfully mapped to the new assembly. The data are available as supplementary files S1 and S2. The majority of markers on both arrays mapped to the same chromosome on both assemblies, with marker order remaining mostly intact. The largest contiguous off-diagonal collection of markers was found on chromosome 16 in CanFam3.1 and on chromosome 34 in Dog10K_Boxer_Tasha_1.0.

\subsection{Analysis of Duplications}

We identified segmental duplications in the Dog10K_Boxer_Tasha_1.0 assembly using two approaches. First, based on assembly self-alignment, we defined segmental duplications as segments at least $1 \mathrm{~kb}$ in length with a sequence identity of $90 \%$ or greater. This identified 5730 intervals encompassing $28.7 \mathrm{Mb}$ of sequence on the primary chromosome assemblies (Table S3). Second, we identified 321 intervals encompassing $38.3 \mathrm{Mb}$ of sequence based on excess depth of coverage from Illumina sequencing reads. These measures of duplication content are both less than that found in the Great Dane Zoey or CanFam3.1 assemblies [8], indicating that these duplicated sequences are not correctly resolved in the Dog10K_Boxer_Tasha_1.0 genome assembly.

\subsection{Analysis of Repetitive Sequences}

We identified common repeats in the Dog10K_Boxer_Tasha_1.0 assembly using RepeatMasker. A total of $41.1 \%$ of the assembly is comprised of repeats, with most falling into one of three categories: LINEs (469 Mb), SINEs (241 Mb) and LTRs (110 Mb). A complete summary of the repeat element composition is available in Table 4 . We compared the results with an equivalent annotation of CanFam3.1. As before, we limited analyses to the primary chromosome sequences. At a high level, the repeat content of the Dog10K_Boxer_Tasha_1.0 and CanFam3.1 assemblies is similar (Table 4). However, the primary chromosome sequences in the Dog10K_Boxer_Tasha_1.0 assembly includes substantially more sequence classified as 'satellite', reflecting the ability of long-read sequencing to extend into subtelomeric and pericentromeric chromosomal regions. Although RepeatMasker analysis indicates that the CanFam3.1 contains more sequence classified as both LINE and SINE than the Dog10K_Boxer_Tasha_1.0 assembly, closer analysis revealed unexpected differences in repeat content (Table 5). LINE and SINE retrotransposons move via a copy-and-paste mechanism and new insertions accumulate mutations over evolutionary time scales [39]. Focusing on the youngest sequences shows that CanFam3.1 contains over 9000 more copies of a family of carnivore SINEs (SINECs) that show less than $10 \%$ sequence divergence, while the Dog10K_Boxer_Tasha_1.0 assembly contains 576 more LINEs that have less than $10 \%$ sequence divergence and are longer than 
$4 \mathrm{~kb}$. We aligned the Dog10K_Boxer_Tasha_1.0 and CanFam3.1 assemblies to further explore this difference in the content of SINEC and LINE elements that have a low sequence divergence and identified 55,329 insertion-deletion differences between the assemblies longer than $10 \mathrm{bp}$. The variant size distribution has clear peaks corresponding to the expected sizes of dimorphic LINEs and SINEs (Figure 2).
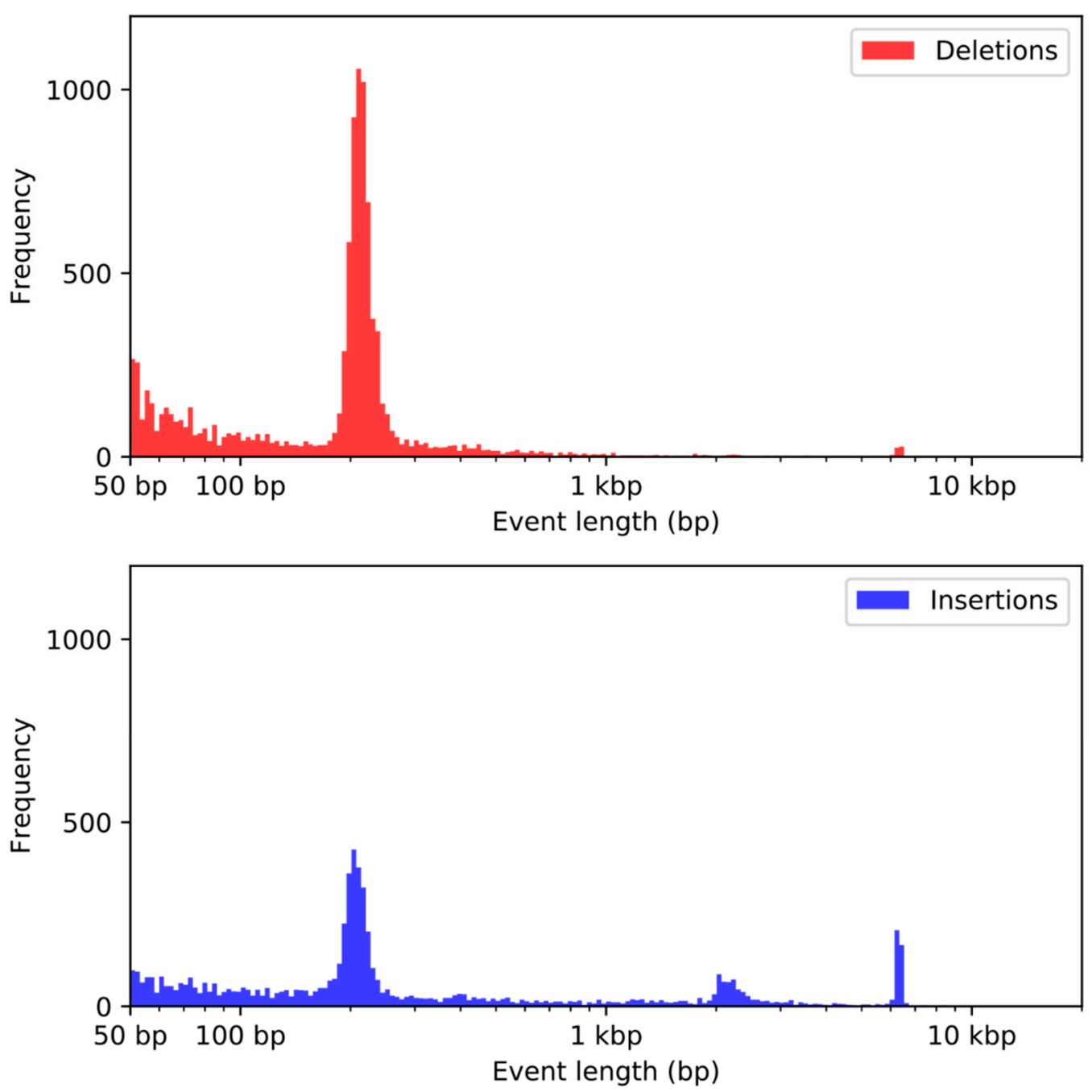

Figure 2. Size distribution of insertion-deletion differences identified between the Dog10K_Boxer_Tasha_1.0 and CanFam3.1 assemblies. The sizes of 22,330 sequences present in CanFam3.1 but absent in Dog10K_Boxer_Tasha_1.0 (red, deletions) and of 32,999 sequences present in Dog10K_Boxer_Tasha_1.0 but absent in CanFam3.1 (blue, insertions) are shown. The bins of each histogram are of equal size on a logarithmic scale.

Since LINE and SINE insertions are highly polymorphic among canines [8,40], we reasoned that the representation in the Dog10K_Boxer_Tasha_1.0 and CanFam3.1 assemblies may reflect the differential inclusion of heterozygous insertions. To assess this possibility, we identified structural variants relative to each assembly using the Tasha PacBio reads. Given the challenges associated with accurately discovering large insertions, we focused our analysis on deletion variants. We identified 35,187 deletions based on alignment to CanFam3.1 and 26,667 deletions based on alignment to Dog10K_Boxer_Tasha_1.0 (Supporting Files S3 and S4). Analysis of the variant size distribution is consistent with differential representation of heterozygous SINEs and LINEs in the two assemblies; there is an excess of $\sim 200 \mathrm{bp}$ deletions when mapping to CanFam3.1, while there is an excess of $\sim 6 \mathrm{~kb}$ deletions when mapping to Dog10K_Boxer_Tasha_1.0 (Figure 3). 

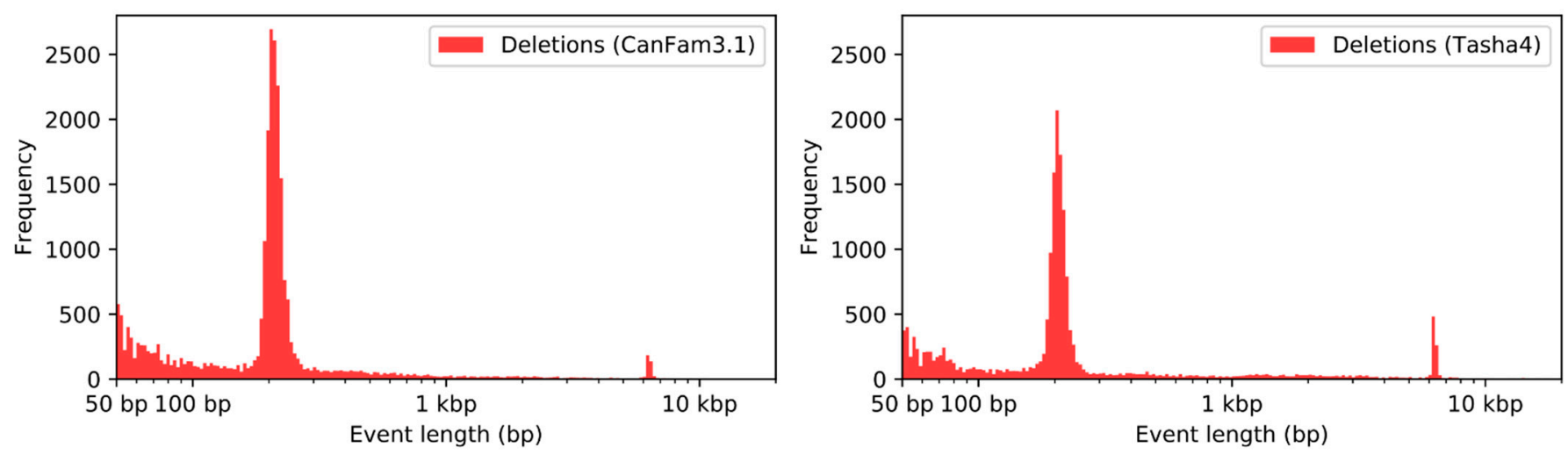

Figure 3. Discovery of deletion variants using PacBio reads. Deletions were identified based on alignment of PacBio reads to the CanFam3.1 (left) or Dog10K_Boxer_Tasha_1.0 (right) assemblies. The bins of each histogram are of equal size on a logarithmic scale.

Table 4. Repeat content of the Dog10K_Boxer_Tasha_1.0 and CanFam3.1 assemblies. Results are shown for the primary chromosome sequences.

\begin{tabular}{ccccc}
\hline & Dog10K_Boxer_Tasha_1.0 & \multicolumn{2}{c}{ CanFam3.1 } \\
\hline Repeat Class & Elements & bp & Elements & bp \\
\hline DNA & 341,866 & $65,043,282$ & 347,025 & $65,997,048$ \\
\hline LINE & $1,286,663$ & $467,394,285$ & $1,307,498$ & $470,518,469$ \\
\hline LTR & 378,505 & $111,520,139$ & 384,551 & $113,151,392$ \\
\hline Low_complexity & 123,075 & $6,525,287$ & 120,803 & $6,009,804$ \\
\hline RC & 1636 & 345,889 & 1649 & 347,342 \\
\hline RNA & 489 & 103,097 & 504 & 105,770 \\
\hline SINE & $1,579,792$ & $240,791,186$ & $1,605,511$ & $244,461,861$ \\
\hline Satellite & 5730 & $11,298,647$ & 635 & 624,881 \\
\hline Simple_repeat & 891,331 & $40,450,974$ & 895,091 & $38,358,719$ \\
\hline Unknown & 3449 & 559,562 & 3487 & 565,722 \\
\hline rRNA & 953 & 129,078 & 958 & 115,711 \\
\hline scRNA & 70 & 4996 & 71 & 5156 \\
\hline snRNA & 4492 & 278,022 & 4617 & 285,578 \\
\hline srpRNA & 45 & 8900 & 47 & 9496 \\
\hline tRNA & 35,501 & $2,608,084$ & 35,906 & $2,636,278$ \\
\hline
\end{tabular}

Table 5. Repeat content for the lowly diverged SINE and LINE sequences.

\begin{tabular}{ccccc}
\hline & \multicolumn{2}{c}{ Dog10K_Boxer_Tasha_1.0 } & \multicolumn{2}{c}{ CanFam3.1 } \\
\hline Repeat Class & Elements & bp & Elements & bp \\
\hline SINEC & $1,125,416$ & $177,104,238$ & $1,146,663$ & $180,147,553$ \\
\hline SINEC $<10 \%$ divergence & 454,869 & $71,490,885$ & 464,113 & $72,819,234$ \\
\hline LINE/L1 & 853,212 & $379,452,954$ & 869,259 & $381,738,114$ \\
\hline $\begin{array}{c}\text { LINE/L1 }<10 \% \text { divergence } \\
\text { and } \geq 4 \mathrm{~kb}\end{array}$ & 4805 & $26,935,018$ & 4229 & $23,359,516$ \\
\hline
\end{tabular}

\subsection{Duplications at the Pancreatic Amylase Locus}

Pancreatic amylase (AMY2B) catalyzes starch to maltose sugar in the small intestine. Changes in amylase gene copy number and expression have been correlated with dietary preferences across mammals [41]. Carnivores such as wolf, coyote and golden jackal have 
two copies of the gene [42,43]. Increased copy number of the gene AMY2B, has been associated with adaptation to a starch-rich diet in modern dogs [42,44,45]. AMY2B copy number is variable both within and among modern dog breeds [46], suggesting a dynamic copy number state, perhaps reflecting recurrent expansion and contraction of a tandemly duplicated array. Long-read assembly data from a Basenji, named China [9], and a German Shepherd, named Nala [47], support the presence of a tandemly duplicated architecture at the AMY2B locus. In addition to tandem duplications, large segmental duplications encompassing AMY2B have also been described [26,48].

In the Dog10K_Boxer_Tasha_1.0 assembly, $A M Y 2 B$ is represented as a single copy on chromosome 6. Using Illumina read data, we estimate that the diploid AMY2 copy number in Tasha is 12 (Figure 4). We found that Tasha is also heterozygous for a large duplication encompassing this locus. Examination of aligned fosmid end-sequence pairs revealed two clusters of clones that have an everted orientation consistent with a tandem duplication structure [49]. We identified the boundaries of these tandem duplications using the raw PacBio reads, defining the boundaries of tandem duplication units that are 1.9 $\mathrm{Mb}$ and $14.9 \mathrm{~kb}$ in length. Due to the presence of the larger duplication, the $12 \mathrm{AMY} 2 \mathrm{~B}$ copies found in the Dog10K_Boxer_Tasha_1.0 genome are distributed among three structural alleles. Using Nanopore sequencing, we assembled a BAC mapping to this locus (CH82-451P03), and found that it contains a single copy of the $A M Y 2 B$ gene (Figure S6). Thus, at least one of the three structural $A M Y 2 B$ alleles in Tasha contains a single copy of this gene.

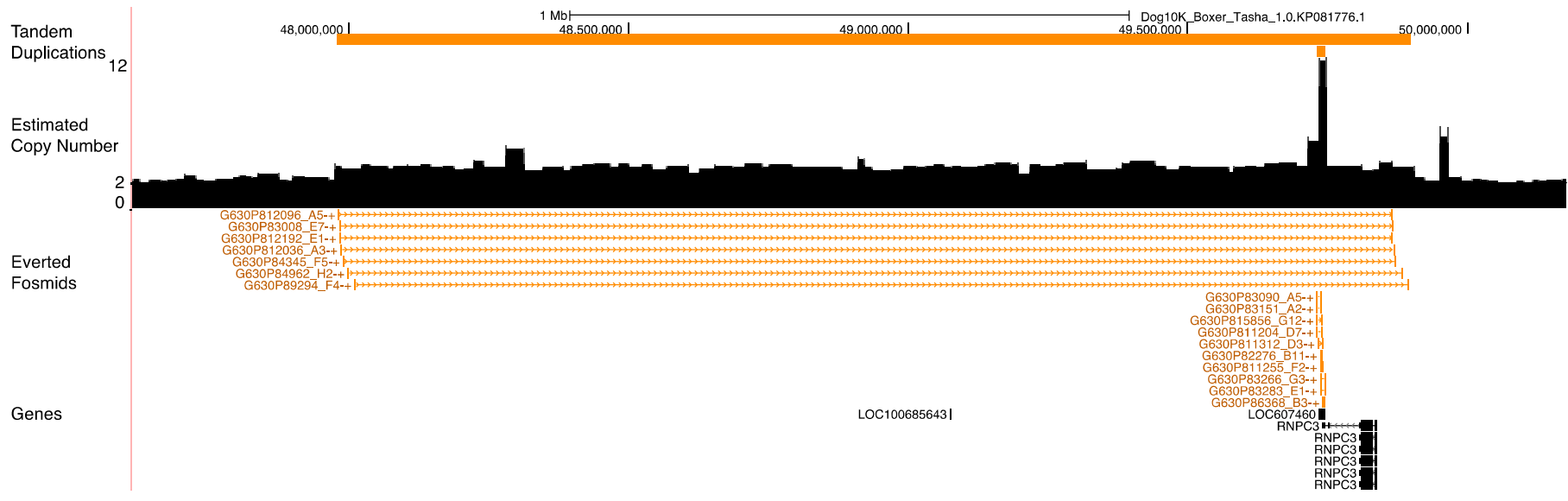

Figure 4. Structural variation at the amylase locus. A genome browser view illustrating structural variation at the amylase locus in Tasha is shown. The orange bars at the top indicate the locations of tandem duplications identified using the raw PacBio long-read data. This includes a large, 1.9 Mbp duplication (chr6:47977592-49898283) as well as a $14.8 \mathrm{kbp}$ duplication (chr6:49729008-49743863). A read depth profile showing copy number estimated from Illumina sequencing data is depicted as a bar plot across the interval. An elevated copy number of 3, corresponding to the $1.9 \mathrm{Mb}$ duplication, is observed, as well as a spike in copy number overlapping with the $A M Y 2 B$ gene. Mappings of discordant fosmid end sequences are shown in orange below the copy number profile. Each depicted clone has end sequences that align in an everted orientation consistent with the presence of a tandem duplication. The position of gene models derived from the NCBI gene annotation, release 106, are shown at the bottom of the figure. The LOC607460 gene model corresponds to pancreatic $\alpha$-amylase $(A M Y 2 B)$.

\section{Discussion}

Canis lupus familiaris, the domestic dog, is now well-established as a genetic system for studies of disease susceptibility, physiology and morphology, all of which inform our understanding of human health. Major advances in human disease genetics have resulted directly from observations made in the dog. Some prominent examples include the identification of PNPLA1 variants in human patients with autosomal recessive congenital ichthyosis 10 that was enabled by results obtained in Golden Retrievers [50] or the elucidation of the role of the $P R C D$ gene in dogs with progressive cone-rod dystrophy and human patients with retinitis pigmentosa [51]. In addition, because of the availability of a canine 
genome assembly, canine disease models are now well established for several diseases including Duchenne muscular dystrophy [52], hypohidrotic ectodermal dysplasia [53], and Leber congenital amaurosis [53]. Similarly, canid evolution has revealed new insights into shifts in canine behaviors that are both surprising and informative, and evinced human dependence on dogs for early survival. While early canine studies relied on segregation studies in families, and later, GWAS studies in case-control cohorts, the most informative studies now rely on large numbers of SNVs and small indels retrieved from publicly available sequences aligned to the reference genome. As such, the reference genome is of critical importance, as current sequence-based GWAS studies highlight not just gene regions, but genic or regulatory variants of interest.

Using PacBio and 10x Chromium long-reads, Dog10k_Boxer_Tasha_1.0 was generated as a new dog genome resource, with a dramatically increased continuity. CanFam3.1 had a contig size of only $267 \mathrm{~kb}$, while the Dog10k_Boxer_Tasha_1.0 assembly has an N50 contig size of $27.3 \mathrm{Mb}$ featuring a $>100$-fold increase in sequence continuity. The improvements in the Dog10k_Boxer_Tasha_1.0 genome sequence relative to the CanFam3.1 assembly included not only greater continuity and fewer gaps, but also led to the correction of misassembled gene regions such as OCA2 (Figure S4), which were supported by concordant alignments of BAC end sequences to the Dog10k_Boxer_Tasha_1.0 assembly.

The improvements in continuity and quality yielded a stronger template for annotation, resulting in better gene models. There is a $7.0 \%$ increase in protein-coding genes with high-coverage ( $\geq 90 \%$ ) alignments in SwissProt, likely resulting from the increased contiguity, and the percentage of protein-coding genes annotated with corrections for suspected frameshifts or premature stop codons is the lowest of any current canine assembly (4.5\%, vs. 5.6-11.3\%), which may reflect the use of CLR reads and an additional polishing step. There are 78 of 2743 known RefSeq transcripts $(2.8 \%)$ that do not map to the Dog10k_Boxer_Tasha_1.0 assembly, which is higher than observed for other assemblies and for which we cannot rule out transcript sequence characteristics or undetected chromosomal deletions, which requires further investigation. In particular, whole genome alignments between Dog10k_Boxer_Tasha_1.0 and previous Tasha assemblies highlight two major deletions on the $\mathrm{X}$ chromosome in the new assembly: an $8 \mathrm{Mb}$ deletion (NC_051843.1: 14.2M..22.2M) and a 4.5 Mb deletion (NC_051843.1: 72M..76.5M). Additional sequencing of the $\mathrm{X}$ chromosome is required to resolve these regions.

There is a systematic underrepresentation of GC-rich sequences in CanFam3.1, as the necessary cloning and sequencing steps did not amplify GC-rich DNA particularly well. Long-read sequencing for the new assembly did not use any cloning steps or PCR and, as a result, GC-rich sequences are better represented and many gaps that were present in CanFam3.1 could be closed. This is critical as GC-rich sequences are often found in the first exons and promoter regions of genes, and play important roles in regulation, such as through differential methylation of $\mathrm{CpG}$ islands. As a result, the Dog10k_Boxer_Tasha_1.0 assembly will allow for more accurate identification of genetic variation in GC-rich regulatory regions and methylome studies.

Since the assembly approach we employed results in a haploid assembly representation, heterozygous loci are not uniformly represented. Essentially, only a single allele at a heterozygous site is included in the assembly. The effect of the haploid representation is most pronounced at heterozygous sites of structural variation where the two alleles may differ by hundreds or thousands of nucleotides. Intriguingly, the CanFam3.1 and Dog10k_Boxer_Tasha_1.0 assemblies have a systematic difference in the inclusion of alleles for dimorphic SINEC and LINE-1 sequences. Thus, although long-read sequencing approaches can resolve the full sequence of large insertions, genome assemblies that represent a diploid sample as a single haplotype may yield an incomplete representation of the true extent of mobile element diversity in canines.

To date, five long-read-based de novo dog genome assemblies [8-10] have been made available at the NCBI genome repository with comparable parameters such as number of 
genes annotated and number of gaps between the new assemblies. The NCBI has annotated all five genomes and made them available on their genome browser https://www.ncbi.nlm.nih.gov/genome/gdv/?org=canis-lupus-familiaris (accessed on 1st Feb 2021). The comparative results indicate a strong likelihood that more protein-coding transcripts, pseudogenes, and non-coding genes remain to be discovered and annotated. However, the highly continuous genome sequence reported here provides a greatly improved framework which will enhance the characterization of functional sequences, genetic variation, and improve the utility of the thousands of canid sequences already generated, setting the stage for genetic studies of high accuracy and resolution.

The availability of de novo assemblies from different breeds will help to characterize structural variants (SVs), including copy-number variations (CNV), mobile element diversity, chromosomal rearrangements, missing sequences and non-redundant sequences. In all species and, especially in dogs, a single reference genome from one individual is unable to represent the full spectrum of divergent sequences in populations worldwide. Dog genomes vary in gene content, including tandem duplicated genes, CNVs distributed throughout the genome and in repetitive parts of the genome such as transposable elements. By characterizing genetic and structural variation within the canine species, de novo assemblies will better reveal the extensive variation in genome content among canine subpopulations defined by breeds, clades, and geography. The extensive analysis of the genetic variability of the canine genome will constitute the next paradigm shift for canine genomics.

\section{Conclusions}

We provide the Dog10k_Boxer_Tasha_1.0 genome assembly derived from the female boxer Tasha, the same dog that was used for the previous genome assemblies CanFam1, 2 and 3. Our assembly represents a substantial improvement in continuity and completeness and, together with the associated annotation, will be a valuable resource for canine and comparative genetics research.

Supplementary Materials: The following are available online at www.mdpi.com/20734425/12/6/847/s1. Figure S1. Apparent fosmid library insert size; Figure S2. Coverage of concordant fosmid clones; Figure S3 Apparent sequence errors based on finished BAC clones; Figure S4. Corrected misassembled regions; Figure S5 Filled Gaps in exons; Figure S6 Alignment of CH82-451P03 to the Dog_10K_Boxer_Tasha_1.0 assembly; TableS1 Regions with no concordant fosmid coverage; Table S2 BACalignedStats.xlsx; TableS3 SegDup-align-table; File S1 Axiom Canine HD Array map file for 10k_Boxer_Tasha; File S2 CanineHD BeadChip map file for 10k_Boxer_Tasha.

Author Contributions: Conceptualization, E.A.O., Y.-P.Z. and G.-D.W.; methodology, V.J., J.M.K., S.E., C.H., B.D.; sequencing: G.-D.W., S.E., J.M.K.; software, V.J. and J.M.K.; Whole genome gene annotation, P.M.; gene annotation analysis, T.D.M.; formal analysis, V.J., J.M.K., C.H., Y.-H.L., R.M.B. and X.-Q.Z.; resources, E.A.O., Y.-P.Z. and G.-D.W.; writing-original draft preparation, V.J., T.L., J.M.K. and E.A.O.; writing - review and editing, V.J., T.L., J.M.K., E.A.O., G.-D.W.; funding acquisition, E.A.O. and Y.-P.Z. All authors have read and agreed to the published version of the manuscript.

Funding: E.A.O. was funded by the Intramural Program of the National Human Genome Research Institute. J.M.K and S.E. were supported by grant R01GM140135 from the National Institutes of Health, The National Key R\&D Program of China (2019YFA0707101), Key Research Program of Frontier Sciences of the CAS (ZDBS-LY-SM011), and Innovative Research Team (in Science and Technology) of Yunnan Province (202005AE160012). G.D.W. is supported by the Youth Innovation Promotion Association of CAS. Funding for the NCBI annotation and analysis, to P.M. and T.D.M., was provided by the Intramural Research Program of the National Library of Medicine, National Institutes of Health.

Institutional Review Board Statement: Not applicable.

Informed Consent Statement: Not applicable. 
Data Availability Statement: The genome assembly is deposited at NCBI under accession number GCF_000002285.5. The associated BioProject accession number is PRJNA13179. BAC clone sequence has been deposited under accession MW972226. UCSC Track hub for Dog_10K_Boxer_Tasha_1.0 assembly https://github.com/KiddLab/tasha_genome_hub (accessed on 29 May 2021).

Conflicts of Interest: The authors declare no conflict of interest.

\section{References}

1. Lindblad-Toh, K.; Wade, C.M.; Mikkelsen, T.S.; Karlsson, E.K.; Jaffe, D.B.; Kamal, M.; Clamp, M.; Chang, J.L.; Kulbokas, E.J.; Zody, M.C.; et al. Genome sequence, comparative analysis and haplotype structure of the domestic dog. Nature 2005, 438, 803819, doi:10.1038/nature04338.

2. Jagannathan, V.; Drögemüller, C.; Leeb, T.; Aguirre, G.; André, C.; Bannasch, D.; Becker, D.; Davis, B.; Ekenstedt, K.; Faller, K.; et al. A comprehensive biomedical variant catalogue based on whole genome sequences of 582 dogs and eight wolves. Anim. Genet. 2019, 50, 695-704, doi:10.1111/age.12834.

3. Plassais, J.; Kim, J.; Davis, B.W.; Karyadi, D.M.; Hogan, A.N.; Harris, A.C.; Decker, B.; Parker, H.G.; Ostrander, E.A. Whole genome sequencing of canids reveals genomic regions under selection and variants influencing morphology. Nat. Commun. 2019, 10, doi:10.1038/s41467-019-09373-w.

4. Xie, X.; Lu, J.; Kulbokas, E.J.; Golub, T.R.; Mootha, V.; Lindblad-Toh, K.; Lander, E.S.; Kellis, M. Systematic discovery of regulatory motifs in human promoters and 3' UTRs by comparison of several mammals. Nature 2005, 434, 338-345, doi:10.1038/nature03441.

5. Dermitzakis, E.T.; Kirkness, E.; Schwarz, S.; Birney, E.; Reymond, A.; Antonarakis, S.E. Comparison of human chromosome 21 conserved nongenic sequences (CNGs) with the mouse and dog genomes shows that their selective constraint is independent of their genic environment. Genome Res. 2004, 14, 852-859, doi:10.1101/gr.1934904.

6. Ramirez, O.; Olalde, I.; Berglund, J.; Lorente-Galdos, B.; Hernandez-Rodriguez, J.; Quilez, J.; Webster, M.T.; Wayne, R.K.; Lalueza-Fox, C.; Vilà, C.; et al. Analysis of structural diversity in wolf-like canids reveals post-domestication variants. BMC Genom. 2014, 15, 465, doi:10.1186/1471-2164-15-465.

7. Serres-Armero, A.; Povolotskaya, I.S.; Quilez, J.; Ramirez, O.; Santpere, G.; Kuderna, L.F.K.; Hernandez-Rodriguez, J.; Fernandez-Callejo, M.; Gomez-Sanchez, D.; Freedman, A.H.; et al. Similar genomic proportions of copy number variation within gray wolves and modern dog breeds inferred from whole genome sequencing. BMC Genom. 2017, 18, 977, doi:10.1186/s12864-0174318-x.

8. Halo, J.V.; Pendleton, A.L.; Shen, F.; Doucet, A.J.; Derrien, T.; Hitte, C.; Kirby, L.E.; Myers, B.; Sliwerska, E.; Emery, S.; et al. Long-read assembly of a great dane genome highlights the contribution of GC-rich sequence and mobile elements to canine genomes. Proc Natl Acad Sci USA 2021, 118, doi:10.1073/pnas.2016274118.

9. Edwards, R.J.; Field, M.A.; Ferguson, J.M.; Dudchenko, O.; Keilwagen, J.; Rosen, B.D.; Johnson, G.S.; Rice, E.S.; Hillier, L.D.; Hammond, J.M.; et al. Chromosome-length genome assembly and structural variations of the primal basenji dog (canis lupus familiaris) genome. BMC Genom. 2021, 22, 188, doi:10.1186/s12864-021-07493-6.

10. Wang, C.; Wallerman, O.; Arendt, M.-L.; Sundström, E.; Karlsson, Å.; Nordin, J.; Mäkeläinen, S.; Pielberg, G.R.; Hanson, J.; Ohlsson, Å.; et al. A novel canine reference genome resolves genomic architecture and uncovers transcript complexity. Commun. Biol. 2021, 4, 1-11, doi:10.1038/s42003-021-01698-x.

11. Koren, S.; Walenz, B.P.; Berlin, K.; Miller, J.R.; Bergman, N.H.; Phillippy, A.M. Canu: Scalable and accurate long-read assembly via adaptive $\kappa$-mer weighting and repeat separation. Genome Res. 2017, 27, 722-736, doi:10.1101/gr.215087.116.

12. Ruan, J.; Li, H. Fast and accurate long-read assembly with Wtdbg2. Nat. Methods 2020, 17, 155-158, doi:10.1038/s41592-019-06693 .

13. Jackman, S.D.; Coombe, L.; Chu, J.; Warren, R.L.; Vandervalk, B.P.; Yeo, S.; Xue, Z.; Mohamadi, H.; Bohlmann, J.; Jones, S.J.M.; et al. Tigmint: Correcting assembly errors using linked reads from large molecules. BMC Bioinform. 2018, 19, doi:10.1186/s12859018-2425-6.

14. Sahlin, K.; Chikhi, R.; Arvestad, L. Assembly scaffolding with PE-contaminated mate-pair libraries. Bioinformatics 2016, 32, 19251932, doi:10.1093/bioinformatics/btw064.

15. English, A.C.; Richards, S.; Han, Y.; Wang, M.; Vee, V.; Qu, J.; Qin, X.; Muzny, D.M.; Reid, J.G.; Worley, K.C.; et al. Mind the gap: Upgrading Genomes with pacific biosciences RS long-read sequencing technology. PLoS ONE 2012, 7, doi:10.1371/journal.pone.0047768.

16. Walker, B.J.; Abeel, T.; Shea, T.; Priest, M.; Abouelliel, A.; Sakthikumar, S.; Cuomo, C.A.; Zeng, Q.; Wortman, J.; Young, S.K.; et al. Pilon: An integrated tool for comprehensive microbial variant detection and genome assembly improvement. PLoS ONE 2014, 9, doi:10.1371/journal.pone.0112963.

17. Alonge, M.; Soyk, S.; Ramakrishnan, S.; Wang, X.; Goodwin, S.; Sedlazeck, F.J.; Lippman, Z.B.; Schatz, M.C. RaGOO: Fast and accurate reference-guided scaffolding of draft genomes. Genome Biol. 2019, 20, doi:10.1186/s13059-019-1829-6.

18. Hitte, C.; Madeoy, J.; Kirkness, E.F.; Priat, C.; Lorentzen, T.D.; Senger, F.; Thomas, D.; Derrien, T.; Ramirez, C.; Scott, C.; et al. Facilitating genome navigation: Survey sequencing and dense radiation-hybrid gene mapping. Nat. Rev. Genet. 2005, 6, 643-648, doi:10.1038/nrg1658. 
19. Seppey, M.; Manni, M.; Zdobnov, E.M. BUSCO: Assessing genome assembly and annotation completeness. In Methods in Molecular Biology; Humana Press Inc., 2019; Vol. 1962, pp. 227-245

20. Quinlan, A.R.; Hall, I.M. BEDTools: A flexible suite of utilities for comparing genomic features. Bioinformatics 2010, 26, 841-842, doi:10.1093/bioinformatics/btq033.

21. Li, H. Minimap2: Pairwise alignment for nucleotide sequences. Bioinformatics 2018, 34, 3094-3100, doi:10.1093/bioinformatics/bty191.

22. Rice, P.; Longden, I.; Bleasby, A. EMBOSS: The European molecular biology open software suite. Trends Genet. 2000, 16, 276277, doi:10.1016/s0168-9525(00)02024-2.

23. Hubley, R.; Finn, R.D.; Clements, J.; Eddy, S.R.; Jones, T.A.; Bao, W.; Smit, A.F.A.; Wheeler, T.J. The Dfam database of repetitive DNA families. Nucleic. Acids Res. 2016, 44, D81-D89, doi:10.1093/nar/gkv1272.

24. Bao, W.; Kojima, K.K.; Kohany, O. Repbase update, a database of repetitive elements in eukaryotic genomes. Mob. DNA 2015, 6, 11, doi:10.1186/s13100-015-0041-9.

25. Numanagic, I.; Gökkaya, A.S.; Zhang, L.; Berger, B.; Alkan, C.; Hach, F. Fast characterization of segmental duplications in genome assemblies. Bioinformatics 2018, 34, i706-i714, doi:10.1093/bioinformatics/bty586.

26. Pendleton, A.L.; Shen, F.; Taravella, A.M.; Emery, S.; Veeramah, K.R.; Boyko, A.R.; Kidd, J.M. Comparison of village dog and wolf genomes highlights the role of the neural crest in dog domestication. BMC Biol. 2018, 16, 64, doi:10.1186/s12915-018-05352 .

27. Kent, W.J.; Baertsch, R.; Hinrichs, A.; Miller, W.; Haussler, D. Evolution's cauldron: Duplication, deletion, and rearrangement in the mouse and human genomes. Proc Natl Acad Sci USA 2003, 100, 11484-11489, doi:10.1073/pnas.1932072100.

28. Kuhn, R.M.; Haussler, D.; Kent, W.J. The UCSC genome browser and associated tools. Brief. Bioinform. 2013, 14, 144-161, doi:10.1093/bib/bbs038.

29. Pruitt, K.D.; Tatusova, T.; Brown, G.R.; Maglott, D.R. NCBI reference sequences (RefSeq): Current status, new features and genome annotation policy. Nucleic. Acids Res. 2012, 40, D130-D135, doi:10.1093/nar/gkr1079.

30. Françoise Thibaud-Nissen; Souvorov, A.; Terence, M.; DiCuccio, M.; Kitts, P. The NCBI Handbook [Internet], 2nd ed.; 2013. Available online: https://www.ncbi.nlm.nih.gov/books/NBK143764/ accessed 1 February 2021).

31. Sedlazeck, F.J.; Rescheneder, P.; Smolka, M.; Fang, H.; Nattestad, M.; von Haeseler, A.; Schatz, M.C. Accurate detection of complex structural variations using single-molecule sequencing. Nat. Methods 2018, 15, 461-468, doi:10.1038/s41592-018-0001-7.

32. Vaser, R.; Sović, I.; Nagarajan, N.; Šikić, M. Fast and accurate de novo genome assembly from long uncorrected reads. Genome Res. 2017, 27, 737-746, doi:10.1101/gr.214270.116.

33. Kurtz, S.; Phillippy, A.; Delcher, A.L.; Smoot, M.; Shumway, M.; Antonescu, C.; Salzberg, S.L. Versatile and open software for comparing large genomes. Genome Biol. 2004, 5, R12, doi:10.1186/gb-2004-5-2-r12.

34. Kent, W.J. BLAT - the BLAST-like Alignment Tool. Genome Res. 2002, 12, 656-664, doi:10.1101/gr.229202.

35. Ewing, B.; Green, P. Base-calling of automated sequencer traces using phred. II. Error probabilities. Genome Res. 1998, 8, 186194.

36. Campbell, C.L.; Bhérer, C.; Morrow, B.E.; Boyko, A.R.; Auton, A. A Pedigree-based map of recombination in the domestic dog genome. G3 Genes Genomes Genet. 2016, 6, 3517-3524, doi:10.1534/g3.116.034678.

37. Wong, A.K.; Ruhe, A.L.; Dumont, B.L.; Robertson, K.R.; Guerrero, G.; Shull, S.M.; Ziegle, J.S.; Millon, L.V.; Broman, K.W.; Payseur, B.A.; et al. A comprehensive linkage map of the dog genome. Genetics 2010, 184, 595-605, doi:10.1534/genetics.109.106831.

38. Canis Lupus Annotation Report. Available online: https://www.ncbi.nlm.nih.gov/genome/annotation_euk/Canis_lupus_familiaris/106/\#TranscriptAlignmentStats (accessed on 15 April 2021).

39. Richardson, S.R.; Doucet, A.J.; Kopera, H.C.; Moldovan, J.B.; Garcia-Perez, J.L.; Moran, J.V. The influence of LINE-1 and SINE retrotransposons on mammalian genomes. Microbiol. Spectr. 2015, 3. doi:10.1128/microbiolspec.MDNA3-0061-2014.

40. Wang, W.; Kirkness, E.F. Short Interspersed elements (SINEs) are a major source of canine genomic diversity. Genome Res. 2005, 15, 1798-1808, doi:10.1101/gr.3765505.

41. Pajic, P.; Pavlidis, P.; Dean, K.; Neznanova, L.; Romano, R.-A.; Garneau, D.; Daugherity, E.; Globig, A.; Ruhl, S.; Gokcumen, O. Independent amylase gene copy number bursts correlate with dietary preferences in mammals. Elife 2019, 8, doi:10.7554/eLife.44628.

42. Axelsson, E.; Ratnakumar, A.; Arendt, M.-L.; Maqbool, K.; Webster, M.T.; Perloski, M.; Liberg, O.; Arnemo, J.M.; Hedhammar, A.; Lindblad-Toh, K. The genomic signature of dog domestication reveals adaptation to a starch-rich diet. Nature 2013, 495, 360364, doi:10.1038/nature11837.

43. Freedman, A.H.; Gronau, I.; Schweizer, R.M.; Vecchyo, D.O.-D.; Han, E.; Silva, P.M.; Galaverni, M.; Fan, Z.; Marx, P.; LorenteGaldos, B.; et al. Genome sequencing highlights the dynamic early history of dogs. PLOS Genetics 2014, 10, e1004016, doi:10.1371/journal.pgen.1004016.

44. Ollivier, M.; Tresset, A.; Bastian, F.; Lagoutte, L.; Axelsson, E.; Arendt, M.-L.; Bălăşescu, A.; Marshour, M.; Sablin, M.V.; Salanova, L.; et al. Amy2B copy number variation reveals starch diet adaptations in Ancient European dogs. $R$ Soc. Open Sci. 2016, 3, 160449, doi:10.1098/rsos.160449.

45. Arendt, M.; Fall, T.; Lindblad-Toh, K.; Axelsson, E. Amylase activity is associated with AMY2B copy numbers in dog: Implications for dog domestication, diet and diabetes. Anim. Genet. 2014, 45, 716-722, doi:10.1111/age.12179. 
46. Reiter, T.; Jagoda, E.; Capellini, T.D. Dietary variation and evolution of gene copy number among dog breeds. PLoS ONE 2016, 11, e0148899, doi:10.1371/journal.pone.0148899.

47. Field, M.A.; Rosen, B.D.; Dudchenko, O.; Chan, E.K.F.; Minoche, A.E.; Edwards, R.J.; Barton, K.; Lyons, R.J.; Tuipulotu, D.E.; Hayes, V.M.; et al. Canfam_GSD: De Novo chromosome-length genome assembly of the German Shepherd dog (Canis Lupus Familiaris) using a combination of long reads, optical mapping, and Hi-C. Gigascience 2020, 9, doi:10.1093/gigascience/giaa027.

48. Botigué, L.R.; Song, S.; Scheu, A.; Gopalan, S.; Pendleton, A.L.; Oetjens, M.; Taravella, A.M.; Seregély, T.; Zeeb-Lanz, A.; Arbogast, R.-M.; et al. Ancient european dog genomes reveal continuity since the Early Neolithic. Nat. Commun. 2017, 8, 16082, doi:10.1038/ncomms16082.

49. Cooper, G.M.; Zerr, T.; Kidd, J.M.; Eichler, E.E.; Nickerson, D.A. Systematic assessment of copy number variant detection via genome-wide SNP genotyping. Nat. Genet. 2008, 40, 1199-1203, doi:10.1038/ng.236.

50. Grall, A.; Guaguère, E.; Planchais, S.; Grond, S.; Bourrat, E.; Hausser, I.; Hitte, C.; Le Gallo, M.; Derbois, C.; Kim, G.-J.; et al. PNPLA1 mutations cause autosomal recessive congenital ichthyosis in Golden Retriever dogs and humans. Nat. Genet. 2012, 44, 140-147, doi:10.1038/ng.1056.

51. Zangerl, B.; Goldstein, O.; Philp, A.R.; Lindauer, S.J.P.; Pearce-Kelling, S.E.; Mullins, R.F.; Graphodatsky, A.S.; Ripoll, D.; Felix, J.S.; Stone, E.M.; et al. Identical mutation in a novel retinal gene causes progressive rod-cone degeneration in dogs and retinitis pigmentosa in humans. Genomics 2006, 88, 551-563, doi:10.1016/j.ygeno.2006.07.007.

52. Kornegay, J.N. The Golden Retriever model of duchenne muscular dystrophy. Skelet. Muscle 2017, 7, 9, doi:10.1186/s13395-0170124-z.

53. Margolis, C.A.; Schneider, P.; Huttner, K.; Kirby, N.; Houser, T.P.; Wildman, L.; Grove, G.L.; Schneider, H.; Casal, M.L. Prenatal Treatment of $\mathrm{X}$-linked hypohidrotic ectodermal dysplasia using recombinant ectodysplasin in a canine model. J. Pharmacol. Exp. Ther. 2019, 370, 806-813, doi:10.1124/jpet.118.256040. 